\title{
Effects of Dietary Supplementation with Sea Buckthorn (Hippophae rhamnoides L.) Seed Oil on an Experimental Model of Hypertensive Retinopathy in Wistar Rats
}

\author{
Konstantinos Bouras ${ }^{a} \quad$ Konstantinos Kopsidas $^{a} \quad$ Michael Bariotakis $^{b}$ \\ Paraskevi Kitsiou $^{c}$ Katerina Kapodistria ${ }^{c}$ Giorgos Agrogiannis ${ }^{d}$ \\ Ioannis Vergados ${ }^{\mathrm{e}}$ Panagiotis Theodossiadis ${ }^{\mathrm{e}}$ Despoina Perrea $^{\mathrm{a}}$ \\ a Laboratory for Experimental Surgery and Surgical Research, Athens University Medical \\ School, Athens, ${ }^{b}$ Department of Biology, University of Crete, Heraklion, ' Institute of \\ Biosciences and Applications, National Centre for Scientific Research, N.C.S.R. "Demokritos," \\ Attiki, d Department of Pathology, University of Athens Medical School, and e Department of \\ Ophthalmology, Attiko General Hospital of Athens, University of Athens, Athens, Greece
}

\begin{abstract}
What Is It about?
Sea buckthorn (SBT) oil is a rich source of phytosterols, flavonoids, unsaturated fatty acids, and carotenoids, which are known for their antioxidant and neuroprotective activity. In this study, we investigated the neuroprotective and antioxidant effect of SBT oil on rat retinopathy secondary to systemic hypertension. The antiapoptotic effect of SBT was determined by measuring glial fibrillary acidic protein, caspase-3, and glutamine synthetase levels with immunohistochemistry and Western blot. Our findings revealed that nephrectomy and salt intake resulted in an increase in systemic blood pressure. Furthermore, we showed that SBT could notably protect the retina from damage induced by hypertensive retinopathy.
\end{abstract}

\section{Keywords}

Hypertensive retinopathy · Retinal ganglion cells · Oxidative stress $\cdot$ Sea buckthorn

\section{Abstract}

Background/Aims: Sea buckthorn (Hippophae rhamnoides L.) oil is a rich source of phytosterols, flavonoids, unsaturated fatty acids, and carotenoids, known for their antioxidant and neuroprotective activity. In this study, we investigated the neuroprotective and antioxidant effect of sea buckthorn oil on rat retina in hypertensive retinopathy. Methods: Twenty-eight male 6-month-old Wistar rats were separated into 3 groups: (1) controls, (2) unilateral nephrectomized rats receiving drinking water with $1 \% \mathrm{NaCl},(3)$ unilateral nephrectomized rats receiving $0.5 \mathrm{~mL}$ sea buckthorn oil and drinking water with $1 \% \mathrm{NaCl}$. Systemic pressures were 
being measured with the tail-cuff method. The antiapoptotic effect of sea buckthorn was determined by measuring glial fibrillary acidic protein (GFAP), cleaved caspase-3, and glutamine synthetase levels with immunohistochemistry and Western blot. Results: Nephrectomy and salt intake caused increases in both systolic and diastolic pressures. Both types of analysis showed that group 2 had statistically significant increases in the expression of GFAP and cleaved caspase-3, while group 3 showed no significant differences compared with the control group. The expression of glutamine synthetase showed no significant differences between the 3 groups. Conclusions: Our findings suggest that sea buckthorn could notably protect the retina from damage induced by hypertensive retinopathy.

(C) 2017 The Author(s)

Published by S. Karger AG, Basel

\section{Introduction}

Pathological fundal appearance may often be the initial finding in patients with asymptomatic essential systemic hypertension [1]. In the course of hypertensive eye disease, clinical changes may involve the choroid, the optic nerve and the retina [2]. Persistently elevated blood pressure has been associated with precapillary arteriolar damage and subsequent breakdown of the inner blood retinal barrier. Moreover, clinical and histological findings like retinal arteriole hyalinization, thickening of vascular basement membrane, capillary closure, cotton-wool spots, arteriolar wall necrosis, and macular star lipid configuration may be noted $[1,3]$. Some experimental and clinical studies have shown that clinical signs of hypertensive retinopathy improve with the use of pharmaceutical treatment for adequate systemic blood pressure control [4]. However, increased blood pressure alone cannot explain the extent of hypertensive retinopathy, and therefore associations with oxidative stress have been studied and revealed [5]. The exact pathogenesis of the disease is yet to be investigated, and a potential antioxidant treatment might be proven beneficial in the course of its prevention and treatment.

Sea buckthorn (SBT; Hippophae rhamnoides L.) of the Elaeagnaceae family naturally grows in Asia and Europe at a height of 2.5-4.3 m. It has been used in traditional oriental medicine for more than one millennium for the treatment of numerous conditions [6]. The SBT seed oil is one of the most nutritious natural oils as it is rich in bioactive components like tocopherols, carotenoids, flavones, phytosterols, lipids, organic acids, tannins, and vitamins (A, C, E, K, riboflavin, folic acid). Recent studies have boosted interest in its pharmacological activities including antioxidant, anti-atherogenic, hepatoprotective, immunomodulatory, and tissue repair properties $[7,8]$. The benefits of SBT seed oil dietary intake during hypertensive retinopathy have not been examined yet.

The retina contains 2 different sorts of macroglial cells which are the Müller cells and astrocytes. Glial fibrillary acidic protein (GFAP) is normally expressed in astrocytes but not in Müller cells except in the case of neuronal injury or degeneration $[9,10]$. Possible alteration of GFAP expression in hypertensive retinopathy is a very effective biomarker to monitor retinal degeneration as well as beneficial protective effects of SBT dietary intake.

Glutamine synthetase (GS) is an enzyme located mostly in Müller cells and plays an important role in the clearance of extracellular glutamate. Prolonged increase in extracellular glutamate is toxic and associated with retinal ganglion cell death [11]. Therefore, it is another very useful indicator for the determination of various neurodegenerative and neuroprotective effects on rat retina.

Cleaved caspase- 3 is one of the main proteases taking part in the process of neuronal apoptosis. Several experimental studies have revealed that apoptosis is a main contributor to 
cell death during the course of several conditions like age-related macular degeneration, glaucoma, retinitis pigmentosa, pathological myopia and diabetic retinopathy. It has been found that it plays a main role in the programmed cell death of the retinal ganglion cells as well as the photoreceptors during degenerative processes as the ones detailed above [12]. In light of that, it is another essential biomarker for our current research on hypertensive retinopathy.

This study was aimed at investigating the changes in the expression of the biomarkers detailed above in the rat retina during hypertensive retinopathy. Therefore, we organized an experimental model to induce essential hypertension and examined for the first time whether dietary intake of SBT seed oil plays a neuroprotective and antioxidant role in the rat retina in this condition.

\section{Methods}

All experimental procedures in this study were conducted under the permission and guidelines provided by the National Greek Bioethics Committee for the use of experimental animals as well as the Veterinary Committee of the Municipality of Athens.

\section{Animal Model}

We were supplied with 28 6-month-old nonrelated Wistar rats, weighing 380-420 g (EKEFE, Demokritos Institute, Athens, Greece). Rats were housed at 2 per cage and given free access to rat chow and water supply. All rats were kept under controlled temperature $(20 \pm$ $2^{\circ} \mathrm{C}$ ) and relative humidity (50 $\pm 5 \%$ ) with 15 complete air recycles per hour as well as 12 -h light/12-h dark cycle fixed periods every day in the Laboratory of Experimental Surgery and Surgical Research N.S. Christeas (Athens, Greece).

Our study included 3 groups of rats. Group 1 consisted of 8 control Wistar rats which were not nephrectomized, and which did not receive any $\mathrm{NaCl}$ or SBT oil supply. Group 2 consisted of 10 Wistar rats which underwent unilateral nephrectomy and were receiving $1 \%$ $\mathrm{NaCl}$ in their daily drinking water supply. Group 3 consisted of 10 Wistar rats which were unilaterally nephrectomized and were receiving $0.5 \mathrm{~mL} /$ day SBT oil (Eubias, Superfoods) via the oral pathway and $1 \% \mathrm{NaCl}$ through their daily drinking water intake.

Nephrectomies were conducted under general anesthesia with dense intraperitoneal ketamine/xylazine solution injected in doses varying according to individual body weights. One week after nephrectomy, rats of the second and third groups were started on $\mathrm{NaCl}$ and SBT oil dietary intake accordingly. Confirmation of hypertension induction was accomplished with the use of tail-cuff pressure measurement equipment (CODA, Kent Scientific Corporation). Systemic pressure measurements were repeated on a 10-daily basis. After the course of 7 months, all rats were euthanized with the use of intravenous dense Pentothal solution injection. Then, their eyes were enucleated and the retinas from both eyes were dissected as flattened whole mounts as previously reported [13]. Half of the retinas were placed in Eppendorf tubes and frozen at $-80^{\circ} \mathrm{C}$ for further Western blotting analysis. The rest were kept in neutralized formalin solution for immunohistochemistry analysis.

\section{Antibodies and Reagents}

Mouse monoclonal anti-GFAP antibody (Cat. No. 36700) was purchased from Cell Signaling. Rabbit polyclonal anti-GS (C20) antibody (Cat. No. sc-6640) was purchased from Santa Cruz Biotechnology, Inc. Mouse anti- $\beta$-tubulin monoclonal antibody (Cat. No. T4026) was purchased from Sigma. 


\section{Preparation of Protein Extracts from Retinal Tissue}

Retinas were extracted in Hepes lysis buffer $(100 \mathrm{mmol} / \mathrm{L} \mathrm{NaCl}, 1 \%$ [v/v] Triton-X-100, $0.5 \%[\mathrm{w} / \mathrm{v}]$ sodium deoxycholate, $0.2 \%$ SDS, $\left.2 \mathrm{mmol} / \mathrm{L} \mathrm{Na} \mathrm{N}_{2} \mathrm{EDTA}\right), 10 \mathrm{~mol} / \mathrm{L}$ HEPES buffer [pH 7.5], $1 \times$ PhosSTOP (cocktail of phosphatase inhibitors; Roche), and $1 \times$ protease inhibitors cocktail (Roche), for $45 \mathrm{~min}$ at $4^{\circ} \mathrm{C}$. Insoluble material was removed by centrifugation at $14,000 \mathrm{~g}$ for $15 \mathrm{~min}$, and the supernatant was stored at $-80^{\circ} \mathrm{C}$. Protein concentration was determined by the Bradford colorimetric assay (Pierce) with bovine serum albumin as standard.

\section{Western Blotting Analysis}

Immunoblot analysis of protein extracts was performed as previously described. Briefly, electrophoresis in the presence of SDS was performed on 7.5\% polyacrylamide gels under reducing conditions. The resolved proteins were subsequently electro-transferred to HybondECL nitrocellulose membrane (Amersham). Blots were saturated for $1 \mathrm{~h}$ at room temperature with $5 \%$ nonfat milk in Tris-buffered saline/ $0.1 \%$ Tween 20 and incubated overnight at $4{ }^{\circ} \mathrm{C}$ with the appropriate dilutions of anti-GFAP or anti-GS (C20) antibody in the same buffer without Tween 20. Incubations with peroxidase-conjugated goat anti-rabbit or sheep antimouse immunoglobulins and detection of bound peroxidase activity were carried out as described for the enhanced chemiluminescence blotting detection system (Pierce). To ensure equal amounts of protein loading, the blots were stripped (Restore Western Blot Stripping Buffer, Pierce Cat. No. 21059) and re-probed with anti- $\beta$-tubulin antibody. Digital images were obtained and processed with the Adobe Photoshop CS4 version 11.0 software. Quantification of the optical density of GFAP- or GS-positive bands was performed in digital images using the Image J 1.43u image processing and analysis software (National Institutes of Health) [14].

\section{Immunohistochemistry}

After resection, all the collected tissues were placed into $10 \%$ neutralized formalin solution (HT50-1-1, Sigma-Aldrich, Co), and then they were processed with a graded series of alcohols and xylene, and finally they were incubated into paraffin waxes. Four-micrometerthick sections were cut from these paraffin-embedded tissues, deparaffinized using xylene and rehydrated using a graded series of alcohols. Antigen retrieval was performed using a citrate buffer, $\mathrm{pH} 6.0$, at $90^{\circ} \mathrm{C}$ for 2 cycles of $15 \mathrm{~min}$ each. Endogenous peroxidase activity was blocked by the incubation with $3 \%$ hydrogen peroxide for $5 \mathrm{~min}$. A serum-free protein block (Dako, Glostrup, Denmark) was applied for $30 \mathrm{~min}$, and the slides were incubated overnight at $4{ }^{\circ} \mathrm{C}$ with the following antibodies: rabbit polyclonal against GS (clone ab 73593; Abcam Co, UK) diluted 1:1,000, chicken polyclonal against GFAP (clone ab 4674; Abcam) diluted 1:1,000, and rabbit polyclonal against caspase-3 (clone ab 2302; Abcam) diluted 1:10. A 2-step technique was used for the visualization of the antibodies (Envision K 5007; Dako, Glostrup, Denmark), and diaminobenzidine solution was used as chromogen. The slides were then counterstained with hematoxylin for $5 \mathrm{~min}$ and were coverslipped with an aqueous-based mounting medium. Matching tissues were used as positive controls for each antibody and omission of the primary antibody as a negative control. Eosin-hematoxylin slides were digitized with a digital camera (Nikon DS-2MW; Nikon Corp, Tokyo) attached to a light microscope (Nikon eclipse 80i; Nikon Corp, Tokyo) under $\times 200$ magnification, and the images were stored as high-quality compressed files in jpg format. Image analysis was performed using the Image Pro Plus 5.1 software (Media Cybernetics, MD, USA). Brown diaminobenzidine staining was indicative of immunohistochemical expression and was considered positive, whereas the blue hematoxylin counterstain was considered negative, as previously described. Briefly, 2 parameters were used for each slide: the average intensity levels of brown staining 


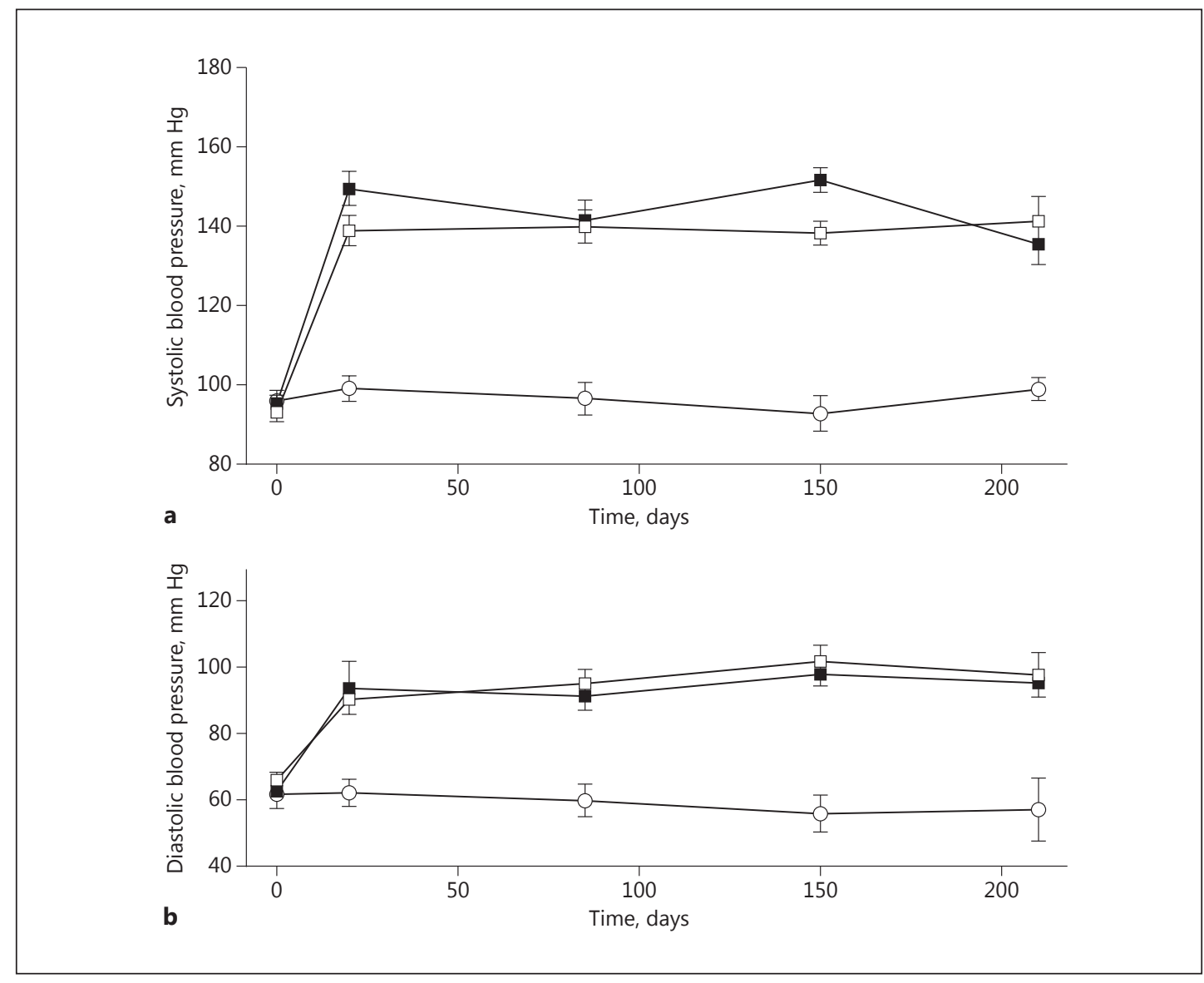

Fig. 1. Mean systolic (a) and diastolic (b) blood pressure of the 3 experimental groups, measured at 0,20 , 85,150 , and 210 days after the nephrectomy. White circles correspond to the control group, white squares correspond to the Hippophae group, and black squares correspond to the hypertensive group. Vertical error bars represent standard error of the mean.

(measured using arbitrary units on a linear scale from 0 [representing black] to 255 [representing white]) and the average percentage of the extent of brown staining. The average values for each slide were calculated as the mean intensity levels of brown staining and the mean percentage of the extent of brown staining [15].

\section{Statistical Analysis}

Statistical analyses were performed using the statistical software R 3.2.2 (R Core Team, Vienna, Austria). Data concerning immunohistochemical expression as well as the Western blotting analysis of proteins GFAP and GS were analyzed by one-way ANOVA, followed by the Tukey HSD test. Data concerning nuclei marked as positive for cleaved caspase- 3 were not normally distributed, so they were analyzed using a nonparametric Kruskal-Wallis test, followed by the nonparametric Mann-Whitney test. Differences were considered significant when $p<0.05$. Unless stated otherwise, results are expressed as mean \pm standard deviation. 

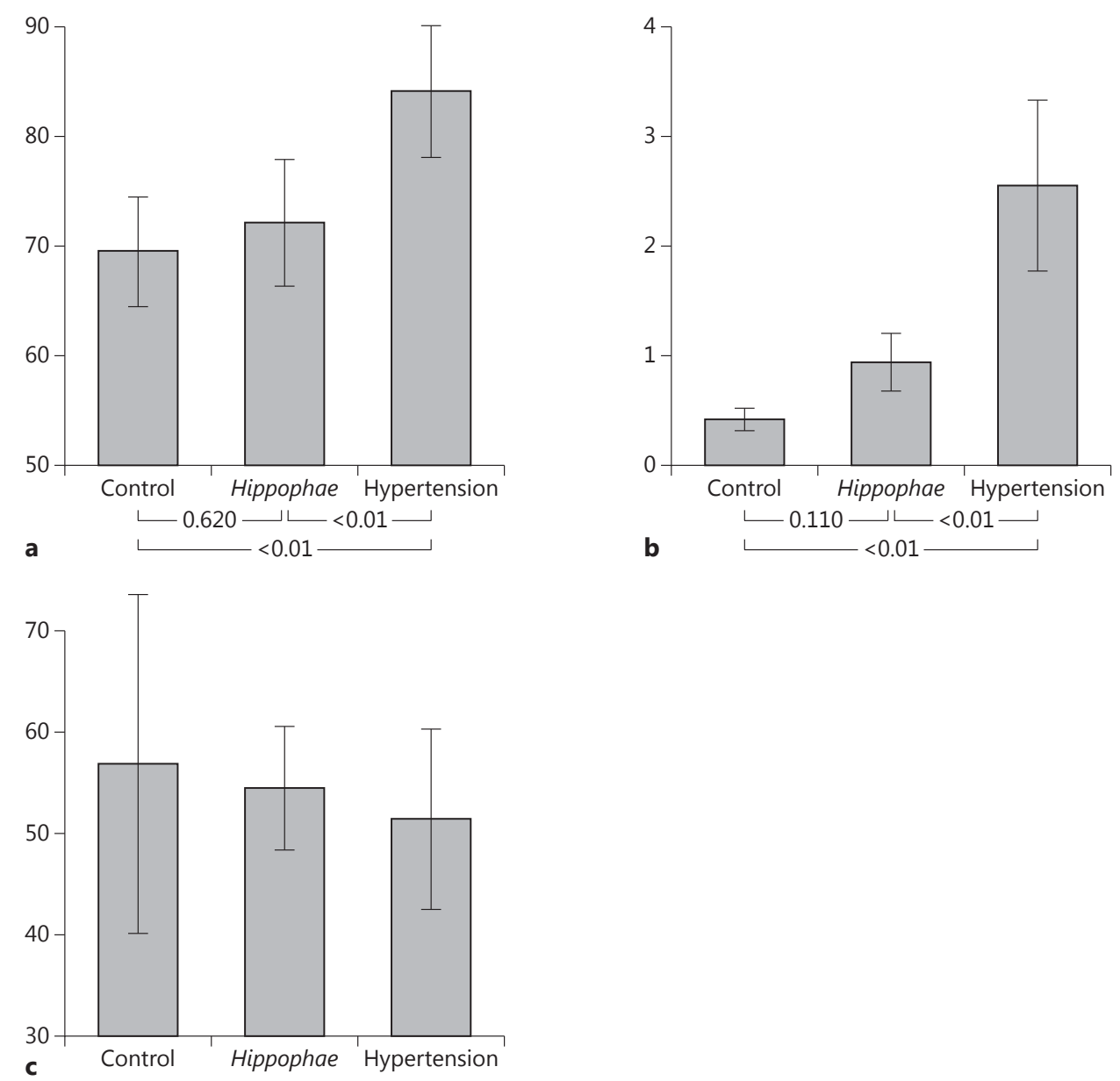

Fig. 2. Results of the immunohistochemical analysis, demonstrating expression of GFAP (a), cleaved caspase-3 (b), and GS (c) for the 3 experimental groups. Bar heights correspond to group means, while vertical error bars represent standard deviation. Numbers bellow the group names express the $p$ values of the respective Tukey HSD tests. In the case of GS, differences in means were insignificant according to ANOVA.

\section{Results}

\section{Blood Pressure}

Nephrectomy treatments of the experimental groups resulted in increases in both systolic and diastolic blood pressures (Fig. 1). In particular, after the 20th day of the experiment, the mean systolic blood pressure of the 2 groups where nephrectomy took place was always higher by at least $36.5 \mathrm{~mm} \mathrm{Hg}$, while mean diastolic pressure was always higher by at least $28.2 \mathrm{~mm} \mathrm{Hg}$. Moreover, there was no sign of drop in the blood pressure of the 2 groups treated with nephrectomy during the 210 days of measurements.

\section{Immunohistochemical Analyses}

Results of immunohistochemical analyses are shown in Figure 2. For GFAP, the hypertensive group showed a significant increase in expression $(84 \pm 6)$ compared to the control group (69.5 \pm 5.02$)$, while the hypertensive group (72.13 \pm 5.76 ) had no significant difference 


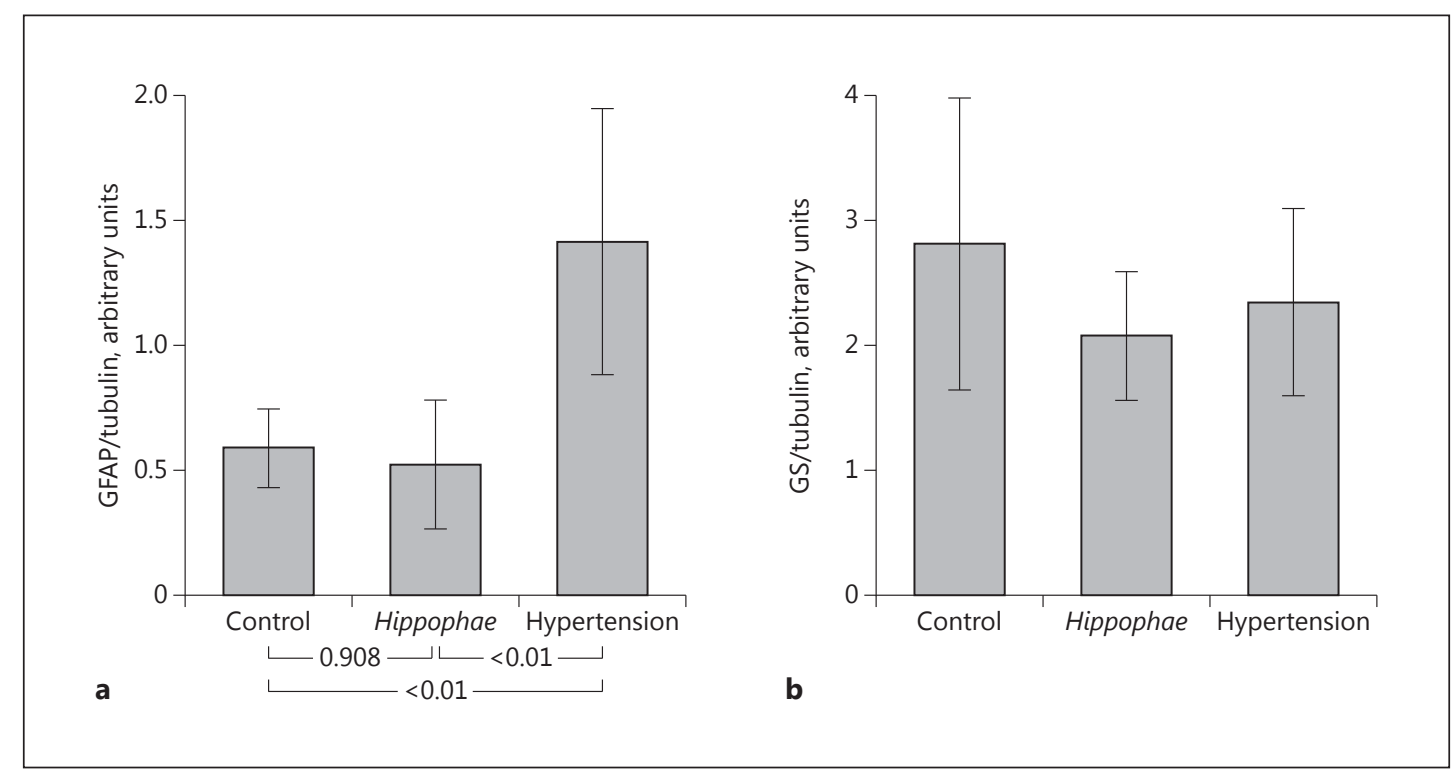

Fig. 3. Quantitative analysis of Western blot for GFAP (a) and GS (b) for the 3 experimental groups. Bar heights correspond to group means, while vertical error bars represent standard deviation. Numbers bellow the group names express the $p$ values of the respective Tukey HSD tests. In the case of GS, differences in means were insignificant according to ANOVA.

Fig. 4. Representative results of GFAP Western blot analysis for the 3 experimental groups.

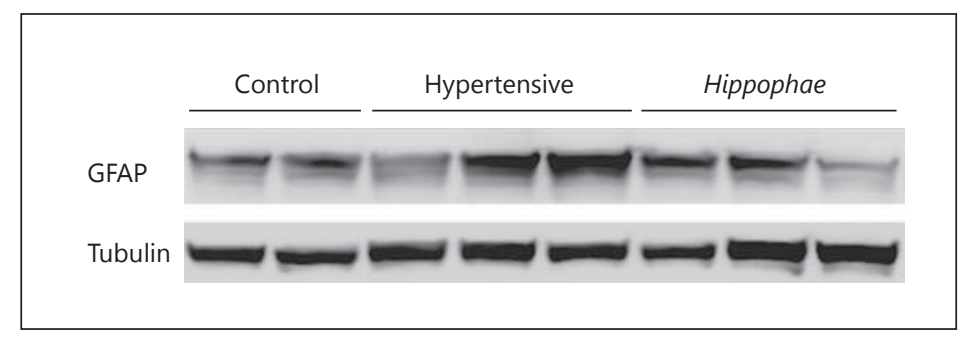

compared with the control group. Cleaved caspase-3 yielded similar results, with the hypertensive group having a significantly higher expression $(2.55 \pm 0.78)$ than the control group $(0.42 \pm 0.1)$, which did not differ significantly from the Hippophae group $(0.94 \pm 0.26)$. The expression of GS, in turn, did not show any significant differences for any of the groups, with values for the control, hypertensive, and Hippophae groups at 56.86 $\pm 16.75,54.47 \pm 6.11$, and $51.42 \pm 8.9$, respectively.

\section{Western Blotting Analyses}

Results of Western blots are in agreement with the above pattern (Fig. 3). Analysis of GFAP showed no significant difference between the control $(0.59 \pm 0.16)$ and Hippophae (0.52 $\pm 0.26)$ groups, while the hypertensive group $(1.31 \pm 0.53)$ had significantly higher levels of GFAP compared to both the control and the Hippophae group (Fig. 4). Once again, the expression of GS showed no significant differences, with the control group yielding values of $2.81 \pm 1.16$, the Hippophae group values of $2.08 \pm 0.51$, and the hypertensive group yielding values of $2.34 \pm 0.75$ (Fig. 5). 
Fig. 5. Representative results of GS Western blot analysis for the 3 experimental groups.
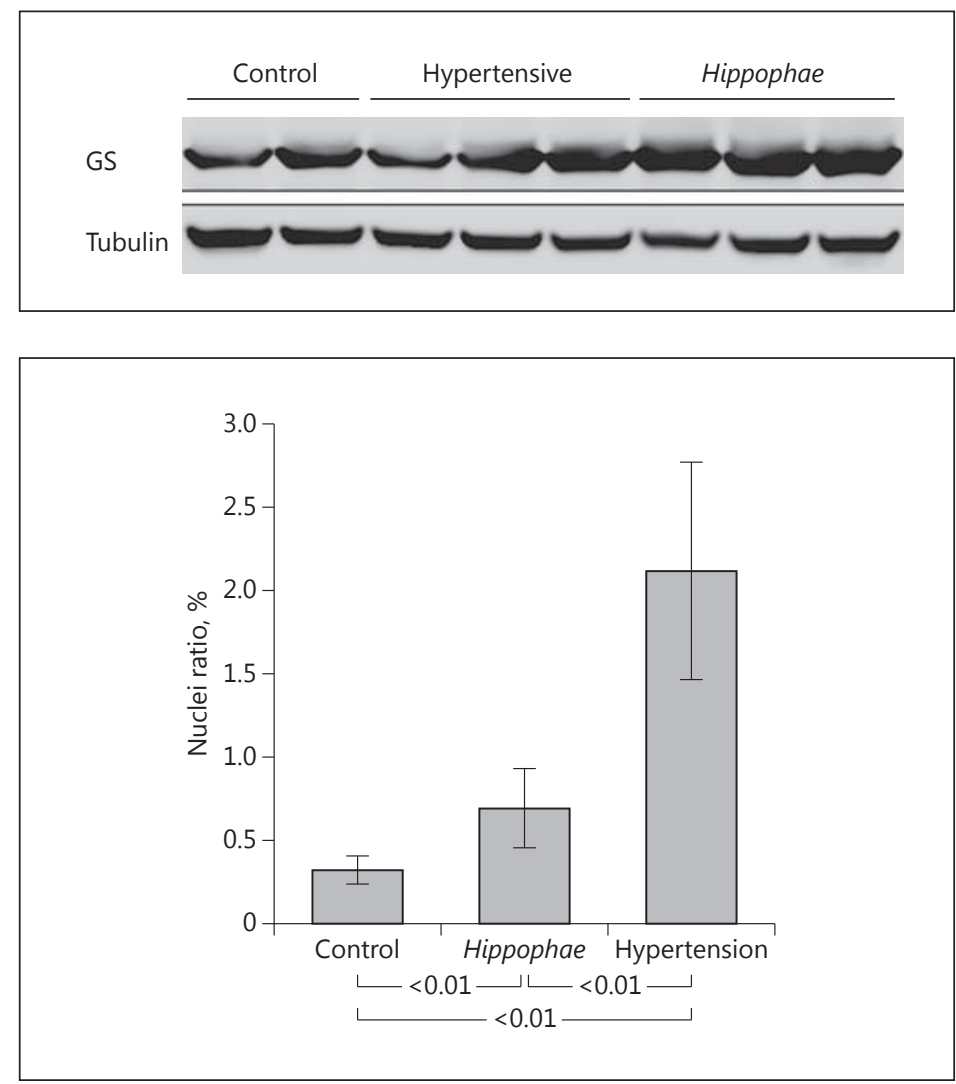

Fig. 6. Comparison of cleaved caspase-3-positive nuclei in the retina of specimens from the 3 experimental groups. Bar heights correspond to group means, while vertical error bars represent standard deviation. Numbers bellow the group names express the $p$ values of the respective Wilcoxon tests.

\section{Nuclei Counts}

The expression of cleaved caspase- 3 in the nuclei of retinal cells shows a gradient in the 3 groups (Fig. 6). Specifically, the control group has the lowest expression $(0.32 \pm 0.8)$, followed by the Hippophae group $(0.69 \pm 0.24)$, while the hypertensive group has the highest expression $(2.12 \pm 0.65)$. All differences between experimental groups were found to be statistically significant.

\section{Localization of Immunostaining}

The 3 immunohistochemical markers were mainly localized in the ganglion cell layer, the nuclear layers, and also the photoreceptor layer. Among them, GS was more diffusely expressed, while cleaved caspase-3 was expressed focally into specific cells. GFAP was also detected in the outer plexiform layer (Fig. 7).

\section{Discussion}

In the present study, we effectively induced systemic hypertension by unilateral nephrectomy and high-dose daily $\mathrm{NaCl}$ consumption. To our knowledge, this is the first study to use this animal model for the investigation of systemic hypertension effects in the retina. Furthermore, it is the first time that the effect of SBT oil dietary intake on the expression of GFAP, GS, and cleaved caspase-3 was investigated in the rat retina during hypertensive retinopathy. Our results suggest that SBT oil consumption plays a neuroprotective and antioxidant role in the rat retina and especially in the ganglion cell layer judging by the inhibition 


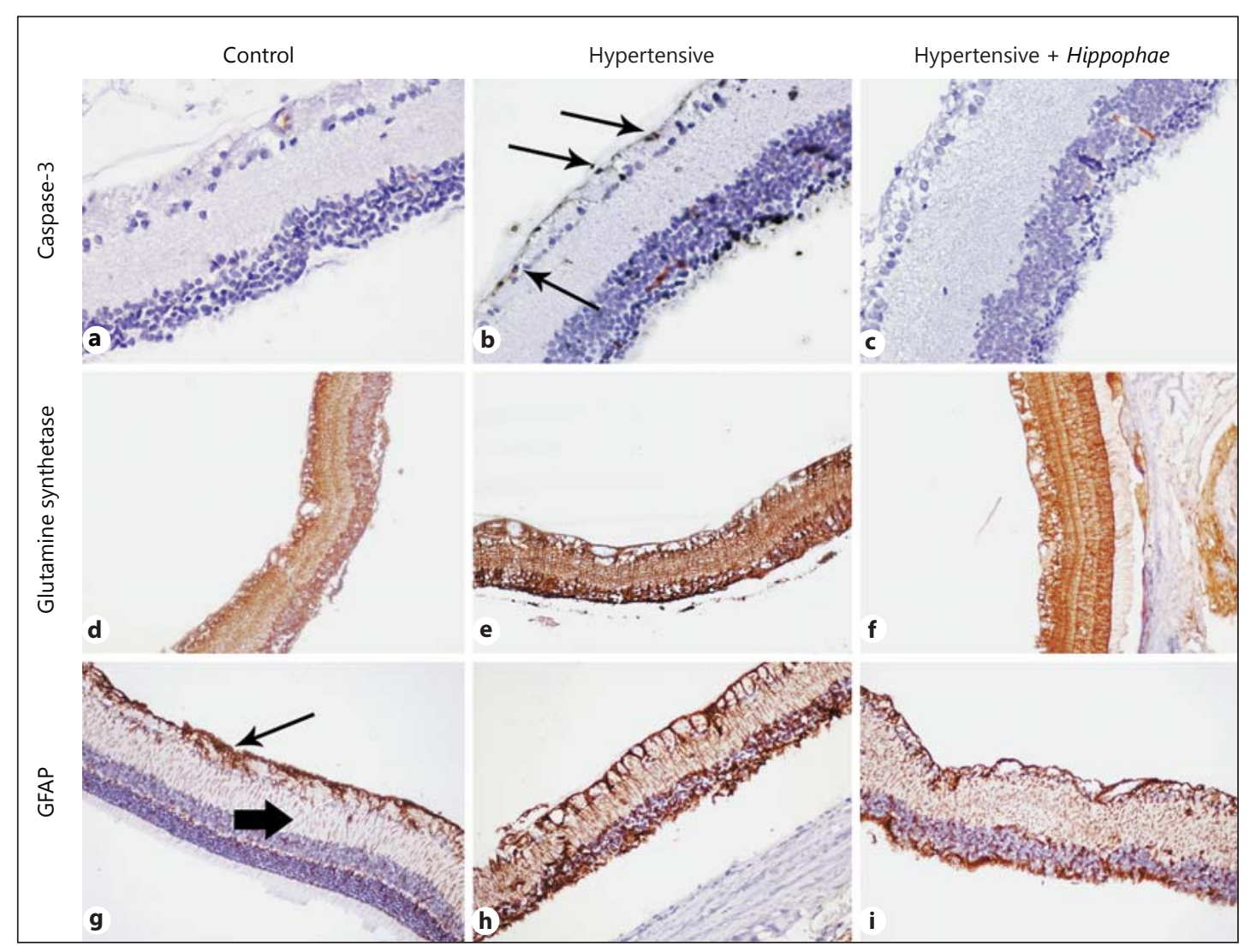

Fig. 7. Representative figures from the experimental groups (columns) and the expression of the immunohistochemical markers (rows) with diaminobenzidine staining in $\times 200$ original magnification. b Apoptotic ganglion cells, as indicated by the expression of cleaved caspase- 3 were more prominent in the hypertensive group (arrows). Glutamine synthetase shows similar expression in all groups (d-f, respectively), while GFAP also showed increased expression in both hypertensive groups. g Arrow shows the expression in ganglion cell layer and arrowhead indicates the expression into the outer plexiform layer.

of cleaved caspase-3 increase. The expression levels of GFAP support the neuroprotective potential of SBT oil in this condition.

Dietary salt loading has been shown to result in systemic blood pressure elevation in various rat experimental models [16-23]. The strongest effect occurs in the genetically selected Dahl salt-sensitive animals, but it also happens in other strains including SpragueDawley rats [17-19]. Other studies have revealed that daily high-salt intake leads to increased arterial blood pressure in dogs and chimpanzees as well $[20,21]$. Systemic hypertension has been caused in rats, rabbits, and chicks by adding 1-2\% NaCl in their drinking water for 9-12 months $[22,23]$. This is the most similar to our experimental model as we replaced their water with $1 \% \mathrm{NaCl}$ aqueous solution for 7 months and effectively caused arterial hypertension in our nephrectomized Wistar rats.

The SBT oil and leaf extract have both been shown to have antioxidant, immunomodulatory, hepatoprotective, antistress, adaptogenic, antibacterial, antiviral, and antiradiation effects in many different animal models so far. The vast majority of the investigations are not in humans, so there is a big margin for research in that aspect. Doses of $15-150 \mathrm{mg} / \mathrm{kg}$ body weight/day have been used variably, so we adjusted the daily intake of our animals accordingly [24]. Inferring from the above, the optimal dose for retinal neuroprotection and anti- 
oxidation is yet to be defined, even though the amount we used revealed beneficial properties in our experimental model.

Previous studies have revealed that nervous tissue damage is accompanied by astrocyte hypertrophy and/or hyperplasia $[25,26]$. There has been a report of an increase in GFAP immunoreactivity in retinal perivascular astrocytes of spontaneous hypertensive rats [26]. Moreover, diabetes has been shown to increase the expression of GFAP in Müller cells while reducing it in astrocytes. Such a reduction has been associated with inability to maintain blood-retinal barrier characteristics in endothelial cells [27]. In the current study, the untreated hypertensive group showed a statistically significant increase in the expression of GFAP. The latter possibly suggests that SBT oil intake prevented retinal astrocyte and Müller cell responses to hypertension, revealing a potential role of SBT oil consumption as a preventive measure against astrocyte hypertrophy and hyperplasia. Consequently, it may act as an effective neuroprotective supplement by helping endothelial cells to maintain their blood retinal barrier characteristics.

GS is the primary glial enzyme responsible for the clearance of extracellular glutamate and plays a critical role in the maintenance of the latter as a neurotransmitter [28]. GS levels have been found to decline in various conditions, such as light damage and retinal detachment, and that is believed to induce ganglion cell loss through NMDA receptor excitotoxicity [29, 30]. Lieth et al. [29] have shown that diabetes gradually reduces glutamate oxidation and glutamine synthesis in the neurosensory retina of experimental rats. Similar GS reactivity has been studied in glaucomatous eyes as well [31,32]. In the current study, the expression of GS did not show any significant differences in any of the groups. This result can be interpreted in 2 different ways. It may suggest that glutamate was not raised during the course of essential hypertension or that SBT oil did not induce neuroprotective increase in GS to maintain normal function of the glutamine/glutamate cycle.

Cleaved caspase- 3 activation has been proven to mediate apoptosis in the inner nuclear and/or ganglion cell layer in a plethora of animal models of retinal degeneration secondary to ischemia, excitotoxicity, and axotomy [33-37]. Furthermore, Wu et al. [38] have demonstrated that procaspase-3 levels are increased following in vivo exposure to blue light leading to cleaved caspase- 3 activation and apoptosis in the photoreceptor layer. An in vitro study by Tezel and Wax [39] has shown cleaved caspase-3 activation in retinal cells induced by various degenerative stimuli. Our study showed that the expression of cleaved caspase- 3 in the control and Hippophae groups was statistically significantly different compared with the hypertensive group. Cleaved caspase- 3 expression was mainly localized in the ganglion cell layer as well as in the photoreceptor cell layer. These results suggest that SBT oil plays a neuroprotective and antiapoptotic role by controlling the activation of cleaved caspase- 3 and concomitantly the apoptotic pathway.

In conclusion, we have demonstrated that daily salt intake induces essential hypertension and concomitant retinal damage in nephrectomized Wistar rats. SBT oil administration showed neuroprotective and antioxidant properties in this case, suggesting its potential role as a prophylactic medicine in the event of retinal damage secondary to arterial hypertension. Taken together, our results provide a framework for further investigations in various retinal degenerative diseases. Additional studies are yet to confirm the full array of beneficial effects of SBT oil.

\section{Disclosure Statement}

No author has a financial or proprietary interest in any material or method mentioned. The authors wish to declare no conflicts of interest. We did not have any funding or sponsorship body supporting our work. 


\section{References}

1 Wong TY, Hubbard LD, Klein R, Marino EK, Kronmal R, Sharrett AR, Siscovick DS, Burke G, Tielsch JM: Retinal microvascular abnormalities and blood pressure in older people. The Cardiovascular Health Study. Br J Ophthalmol 2002;86:1007-1013.

2 Chopra A, Sharma A, Singh S, Bahla V: Changing perspectives in classifications of hypertensive retinopathy. IOSR J Dent Med Sci 2014;13:51-54.

-3 Klein R, Klein BE, Moss SE: The relation of systemic hypertension to changes in the retinal vasculature: The Beaver Dam Eye Study. Trans Am Ophthalmol Soc 1997;95:329-346.

4 Bock KD: Regression of retinal vascular changes by antihypertensive therapy. Hypertension 1984;6:III-158.

5 Karaca M, Coban E, Felek R, Unal M: The association of oxidative stress with hypertensive retinopathy. Clin Exp Hypertens 2013;35:16-19.

6 Upadhyay NK, Kumar R, Mandotra SK, Meena RN, Siddiqui MS, Sawhney RC, Gupta A: Safety and healing efficacy of sea buckthorn (Hippophae rhamnoides L.) seed oil on burn wounds in rats. Food Chem Toxicol 2009; 47:1146-1153.

7 Pang X, Zhao, J, Zhang W, Zhuang X, Wang J, Xu R, Xu Z, Qu W: Antihypertensive effect of total flavones extracted from seed residues of Hippophae rhamnoides L. in sucrose-fed rats. J Ethnopharmacol 2008;117:325-331.

-8 Suryakumar G, Gupta A: Medicinal and therapeutic potential of Sea buckthorn (Hippophae rhamnoides L.). J Ethnopharmacol 2011;138:268-278.

-9 Barber AJ, Antonetti DA, Gardner TW; Penn State Research Group: Altered expression of retinal occludin and glial fibrillary acidic protein in experimental diabetes. Invest Ophthalmol Vis Sci 2000;41:3561-3568.

$\checkmark 10$ Eisenfeld A, Bunt-Milam, AH, Sarthy PV: Müller cell expression of glial fibrillary acidic protein after genetic and experimental photoreceptor degeneration in rat retina. Invest Ophthalmol Vis Sci 1984;251321-1328.

11 Shen F, Chen B, Danias J, Lee KC, Lee H, Su Y, Podos S, Mittag TW: Glutamate-induced glutamine synthetase expression in retinal Müller cells after short term ocular hypertension in the rat. Invest Ophthalmol Vis Sci 2004;45:3107-3112.

-12 Wu J, Gorman A, Zhou X, Sandra C, Chen E: Involvement of caspase-3 in photoreceptor cell apoptosis induced by in vivo blue light exposure. Invest Ophthalmol Vis Sci 2002;43:3349-3354.

13 Salinas-Navarro M, Mayor-Torroglosa S, Jimenez-Lopez M, Aviles-Trigueros M, Holmes TM, Lund RD, VillegasPerez MP, Vidal-Sanz M: A computerized analysis of the entire retinal ganglion cell population and its spatial distribution in adult rats. Vis Res 2009;49:115-126.

14 Kitsiou PV, Tzinia AK, Stetler-Stevenson WG, Michael AF, Fan WW, Zhou B, Tsilibary E: Glucose-induced changes in integrins and matrix-related functions in cultured human glomerular epithelial cells. Am J Renal Physiol 2003;284:671-679.

15 Kostakis ID, Agrogiannis G, Vaiopoulos AG, Mylona G, Patsouris E, Kouraklis G, Koutsilieris M: KISS1 expression in colorectal cancer. APMIS 2013;121:1004-1010.

16 Adeleye OE, Sofola OA: Salt intake and mean arterial blood pressure in rabbits. Sci World J 2010;5:1597-6343.

$>17$ Dahl LK, Heine M, Tassinar L: Effects of chronic excess salt ingestion: evidence that genetic factors play an important role in susceptibility to experimental hypertension. J Exp Med 1962;115:1173-1190.

-18 Giardina JB, Green, GM, Rinewalt AN, Granger JP, Khalil RA: Role of endothelin B receptors in enhancing endothelium-dependent nitric oxide-mediated vascular relaxation during high salt diet. Hypertension 2001;37(2 part 2):516-523.

$\checkmark 19$ Obiefuna PCM, Ebeigbe AB, Sofola OA, Aloamaka PC: Altered responses from aortic smooth muscle from Sprague Dawley rats with salt induced hypertension. Clin Exp Pharmacol Physiol 1991;18:813-818.

20 Haisworth R, Sofola OA, Knill A Drinkhill MJ: Influence of dietary salt on the response of isolated perfused mesenteric artery of the dog to vasoactive agents. Am J Hypertens 2003;16:6-10.

21 Denton D, Weisinger R Mundy N, Wickings EJ, Dixson A, Moisson P, Pingard AM, Shade R, Carey D, Ardaillou R, Paillard F, Chapman J, Thillet J, Michel JB: The effect of increased salt intake on blood pressures of chimpanzees. Nat Med 1995;1:1009-1016.

-22 Boura ALA, Green AF: Antihypertensive agents; in Laurence DR, Bacharach AL (eds): Evaluation of Drug Activities: Pharmacometrics. London, Academic Press, 1964, pp 431-453.

23 Rathod SP, Shah N, Balaraman R: Antihypertensive effect of dietary calcium and diltiazem, a calcium channel blocker on experimentally induced hypertensive rats. Indian J Pharmacol 1997;29:99-104.

-24 Geetha S, Gupta A: Medicinal and therapeutic potential of sea buckthorn (Hippophae rhamnoides L.). J Ethnopharmacol 2011;138:268-278.

25 Latov N, Nilaver G, Zimmerman EA, Johnson WG, Silverman AJ, Defendini R, Cote L: Fibrillary astrocytes proliferate in response to brain injury. Dev Biol 1979;72:381-384.

26 Sabbatini M, Strocchi P, Vitaioli L, Amenta F: Changes of retinal neurons and glial fibrillary acid protein immunoreactive astrocytes in spontaneously hypertensive rats. J Hypertens 2001;19:1861-1869.

27 Barber A, Antonetti D, Gardner T; Penn State Retina Research Group: Altered expression of retinal occludin and glial fibrillary acidic protein in experimental diabetes. Invest Ophthalmol Vis Sci 2000;41:3561-3568.

28 Ehinger B: Glial and neuronal uptake of GABA, glutamic acid, glutamine and glutathione in the rabbit retina. Exp Eye Res 1977;25:221-234.

$\checkmark 29$ Lieth E, Lanque K, Antonetti D, Ratz M, Penn State Retina Research Group: Diabetes reduces glutamate oxidation and glutamine synthesis in the retina. Exp Eye Res 2000;70:723-730. 
-30 Shen F, Chen B, Danias J, Lee K, Lee H, Su Y, Podos S, Mittag T: Glutamate-induced glutamine synthetase expression in retinal Müller cells after short-term ocular hypertension in the rat. Invest Ophthalmol Vis Sci 2004;45:3107-3112.

-31 Dreyer EB, Zurakowski D, Schumer RA, Podos SM, Lipton SA: Elevated glutamate in the vitreous body of humans and monkeys with glaucoma. Arch Ophthalmol 1996;114:299-305.

-32 Brooks DE, Garcia GA, Dreyer EB, Zurakowski D, Franco-Bourland RE: Vitreous body glutamate concentration in dogs with glaucoma. Am J Vet Res 1997;58:864-867.

33 Katai N, Yoshimura N: Apoptotic retinal neuronal death by ischemia-reperfusion is executed by two distinct caspase family proteases. Invest Ophthalmol Vis Sci 1999;40:2697-2705.

34 Kermer P, Klocker N, Labes M, Thomsen S, Srinivasan A, Bahr M: Activation of caspase-3 in axotomized rat retinal ganglion cells in vivo. FEBS Lett 1999;453:361-364.

35 Katai N, Kikuchi T, Shibuki H, et al: Caspaselike proteases activated in apoptotic photoreceptors of Royal College of Surgeons rats. Invest Ophthalmol Vis Sci 1999;40:1802-1807.

-36 Yoshizawa K, Nambu H, Yang J, et al: Mechanisms of photoreceptor cell apoptosis induced by N-methyl-Nnitrosourea in Sprague-Dawley rats. Lab Invest 1999;79:1359-1367.

-37 Liu C, Li Y, Peng M, Laties AM, Wen R: Activation of caspase-3 in the retina of transgenic rats with the rhodopsin mutation s334ter during photoreceptor degeneration. J Neurosci 1999;19:4778-4785.

-38 Wu J, Gorman A, Zhou X, Sandra C, Chen E: Involvement of caspase-3 in photoreceptor cell apoptosis induced by in vivo blue light exposure. Invest Ophthalmol Vis Sci 2002;43:3349-3354.

39 Tezel G, Wax MB: Inhibition of caspase activity in retinal cell apoptosis induced by various stimuli in vitro. Invest Ophthalmol Vis Sci 1999;40:2660-2667. 